\title{
Connectivity in Multi-Channel Multi-Interface Wireless Mesh Networks
}

\author{
Carina Teixeira De Oliveira* Fabrice Theoleyre ${ }^{\dagger}$ Andrzej Duda* \\ ${ }^{*}$ Grenoble Institute of Technology, CNRS Grenoble Informatics Laboratory UMR 5217, France \\ ${ }^{\dagger}$ CNRS, University of Strasbourg, UMR 7005, France \\ Email: \{oliveira,duda\}@imag.fr, theoleyre@unistra.fr
}

\begin{abstract}
We can improve the performance of wireless mesh networks by using multiple interfaces tuned to non-overlapping channels. A Channel and Interface Assignment (CIA) decides when to switch interfaces and which channel to use. Surprisingly, the impact of CIAs on connectivity has received little attention so far. In this paper, we present a comparison and performance evaluation of the existing $C I A$ strategies addressing the connectivity issues: network topology, density of connections, and neighbor discovery. The results presented in this paper provide guidelines for network designers in planning multi-channel multi-interface network deployments.
\end{abstract}

Keywords-wireless mesh network; multi-channel; multiinterface; connectivity; neighbor discovery;

\section{INTRODUCTION}

In this paper, we consider Wireless Mesh Networks (WMNs) with routers based on the IEEE 802.11 technology. When mesh routers use a single interface (i.e. a wireless radio card) tuned to a single channel, the network capacity degrades with the increase of the network size due to channel contention and spatial problems such as hidden and exposed nodes [1].

One way of improving the performance of WMNs is to use multiple non-overlapping channels so that mesh routers can transmit in parallel [2]. However, the operation with multiple channels has to be carefully designed to avoid deafnessthe transmitter must know if the receiver listen to the same channel at the same time. Moreover, nodes may suffer from low connectivity leading to possibly disconnected networks.

To fully take advantage of multiple channels, nodes may have multiple IEEE 802.11 interfaces to simultaneously communicate with many neighbors. Network capacity would increase with the number of interfaces [3]. There are many related papers that study the benefit of using Multi-Channel Multi-Interface (MCMI) in WMN [4]. In spite of considerable similarity, these approaches may adopt different strategies to decide when to switch interfaces as well as which channel to assign. Therefore, the key challenge consists in achieving an effective Channel and Interface Assignment (CIA). The more interfaces assigned to the same channels, the better connectivity. However, interference and contention have oppositely a negative impact on network capacity [5].

Surprisingly, taking into account the impact of CIA on connectivity has received little attention so far. In particular, the problem of network partitions when a network starts up was not well-studied. Similarly, to the best of our knowledge, the impact of CIA strategies on neighbor discovery has never been studied, although it is a major component of network operation.

We propose here to quantify the impact of different strategies on network connectivity and on the neighborhood discovery problem. More precisely, the contribution of this paper is threefold:

1) we study the impact of the channel and interface assignment strategy on the network topology and its connectivity (i.e. the density of the communication graph);

2) we analyze the neighborhood discovery process. We provide in particular an analytical formulation of the expected time before a neighbor is discovered;

3) we provide a formal framework to compare different strategies. This framework can help a WMN designer to choose the most accurate strategy for a given situation (convergence delays versus capacity).

\section{NETWORK MODEL}

We model a WMN as an undirected graph $G=(V, E)$, where $V$ represents the set of nodes in the network and $E$ the set of edges corresponding to two nodes able to directly communicate. The wireless network offers $\mathcal{C}$ orthogonal channels. Each mesh router $v$ is equipped with $I_{v}$ interfaces such as:

$$
\forall v \in V, I_{v}=I_{v}^{S}+I_{v}^{D}
$$

where $I_{v}^{S}$ is the number of Static interfaces and $I_{v}^{D}$ the number of Dynamic interfaces. A Static interface stays tuned to a particular channel, which prevents deafness on reception. A Dynamic interface switches between different channels. It may suffer from deafness on reception-the transmitter must know the channel to use at a given instant.

We consider the situation in which the number of interfaces is smaller than the number of channels: $\forall v \in V, I_{v}<\mathcal{C}$. Otherwise, the assignment problem becomes much simplerwe can assign one interface per channel to maintain a fixed topology.

Two nodes in the radio range of each other can communicate directly if they use the same channel at the same time. More formally, $\forall v_{1}, v_{2} \in V,\left(v_{1}, v_{2}\right) \in E$ if:

$$
\exists i \in \operatorname{Intf}\left(v_{1}\right), \exists j \in \operatorname{Int} f\left(v_{2}\right) / \mathcal{S}(i) \cap \mathcal{S}(j) \neq \emptyset ;
$$

where $\operatorname{Intf}(v)$ is the set of interfaces of node $v \in V$, and $\mathcal{S}(i)$ is the schedule of interface $i-$ a list of tuples $\{($ channels, timeStart, timeStop $)\}$. Moreover, if multiple interfaces at $v_{1}$ and $v_{2}$ share $n$ common channels, there are $n$ 
TABLE I

CIA STRATEgIES

\begin{tabular}{|l|c|c|c|c|c|c|c|}
\hline \multirow{2}{*}{ Strategies } & \multicolumn{2}{|c|}{ Interface Assignment } & \multicolumn{3}{c|}{ Channel Assignment } & \multirow{2}{*}{ References } \\
\cline { 2 - 7 } & Static & Dynamic & Mixed & Common & Pseudo-Random & Adaptive & \\
\hline Static/Common & $\mathrm{X}$ & & & $\mathrm{X}$ & & & {$[6],[7]$} \\
\hline Static/Pseudo-Random & $\mathrm{X}$ & & & & $\mathrm{X}$ & $\mathrm{X}$ & {$[8],[9]$} \\
\hline Dynamic/Adaptive & & $\mathrm{X}$ & & & & {$[10],[11]$} \\
\hline Mixed/Common and Adaptive & & & $\mathrm{X}$ & $\mathrm{X}$ & & $\mathrm{X}$ & {$[12],[13]$} \\
\hline Mixed/Pseudo-Random and Adaptive & & & $\mathrm{X}$ & & $\mathrm{X}$ & $\mathrm{X}$ & {$[14],[15]$} \\
\hline
\end{tabular}

links $\left(v_{1}, v_{2}\right) \in E$, where $n$ is a positive integer: the WMN can be a multigraph.

\section{RELATED WORK}

We introduce here a classification of multi-channel and multi-interface strategies we further use in our analysis.

\section{A. Interface Assignment}

Here, we define the behavior of interfaces in the network.

1) Static Interfaces: all interfaces are static and remain on the same channel all the time $\left(I_{v}=I_{v}^{S}\right)$.

2) Dynamic Interfaces: all interfaces are dynamic and frequently switch from one channel to another $\left(I_{v}=I_{v}^{D}\right)$ It eliminates the channel bottleneck problem, but may suffer from the deafness problem.

3) Mixed Interfaces: $I_{v}^{S}$ static interfaces permanently stay on a channel and $I_{v}^{D}$ dynamic interfaces frequently switch from one channel to another $\left(I_{v}^{S} \geq 1, I_{v}^{D} \geq 1\right)$.

\section{B. Channel Assignment}

Channel assignment decides which channels to assign for both static and dynamic interfaces.

1) Common Channel Set $(C C S)$ : the nodes may agree on using the same (common) channel set for all their static interfaces: the $i^{\text {th }}$ interface uses channel $i$.

2) Pseudo-Random: each node assigns pseudo-randomly a set of channels to its interface.

3) Adaptive: the protocol can dynamically adapt the set of channels used by its interface. Thus, the WMN can proceed with load-aware channel assignment [16] or take into account narrowband interference [17].

\section{Strategies}

A strategy is a combination of interface and channel assignment. Table I presents an overview of existing approaches.

1) Static Interfaces/Common Channel Assignment: this strategy assigns a static channel for each of its interfaces using CCS (the same channel is used for all $i^{\text {th }}$ interfaces) [6], [7]. Thus, two neighbors have always multiple independent links to communicate with each other: this results in a stable network topology without partitions. However, the network capacity decreases when the number of nodes increases as more contention and interference may occur [17].

2) Static Interfaces/Pseudo-Random Channel Assignment: similar to the previous strategy, it assigns a static channel for each interface. However, this assignment is independent between different nodes. Consequently, this strategy does not guarantee connectivity: two nodes may choose different channels for their interfaces leading thus to deafness. We can also color the graph (one channel per interface) [8], [9]. These solutions would preserve the graph connectivity, but require to know the topology and interference.

3) Dynamic Interfaces/Adaptive Channel Assignment: all interfaces are dynamic. Thus, network topology continuously changes. Often, nodes need to use a rendezvous mechanism to avoid deafness: the nodes adopt a schedule such that statistically a pair of node has common timeslots [10]. Nodes can also have their own schedules and adapt them according to the neighborhing schedules like in SSCH [11].

4) Mixed Interfaces/Common and Adaptive Channel Assignment: each node has static interfaces using a CCS, while the dynamic interfaces act in an on-demand manner. For instance, static interfaces may be used to send RTS/CTS and reserve the channel that will be used further by a pair of dynamic interfaces for the data exchange [12], [13].

5) Mixed Interfaces/Pseudo-Random and Adaptive Channel Assignment: the first approach would consist in using static interfaces for reception. Each node randomly chooses the channel used for each of its static interfaces. The transmitter switches one of its dynamic interfaces to one of the static channel of the receiver: no deafness occurs. These strategies often use a single static channel $\left(I_{v}^{S}=1\right)$ [14]. To find the optimal number of static interfaces is a very complex task, leading often to a sub-optimal solution.

Another approach would consist in maximizing the network capacity with static interfaces while dynamic interfaces work in an on-demand manner. In this case, $I_{v}^{D}=1$ [15]. The static interfaces may become a bottleneck.

\section{Connectivity Analysis}

We propose now to quantify the impact of the CIA strategies on the network performance. We will study in particular the following characteristics:

1) Network connectivity: the size of the largest connected component of the multi-channel graph;

2) Density of connections: the ratio of number of radio links that exist respectively in the single channel and multichannel network;

3) Neighbor discovery

a) Probability of Rendezvous $(P(R))$ : the probability of two neighbors selecting at least one common channel among the $\mathcal{C}$ available channels in any time slot $T$. The event of interest is in this case: " $R=$ at least one channel in common".

b) Expected Time to Rendezvous (E[TR]): the expected time to achieve a rendezvous.

We present the results of our evaluation based on a probabilistic analysis, corroborated by simulations. We have implemented a custom-built simulator to evaluate the impact 
TABLE II

DENSITY OF CONNECTIONS - 500 NODES, 3 INTERFACES, 8 CHANNELS

\begin{tabular}{|l|c|}
\hline \multicolumn{1}{|c|}{ Strategies } & Density (\%) \\
\hline Static/Common & 300 \\
\hline Static/Pseudo-Random & 60 \\
\hline Dynamic/Adaptive & 60 \\
\hline Mixed/Common and Adaptive & 110 \\
\hline Mixed/Pseudo-Random and Adaptive & 200 \\
\hline Single Channel CCS & 100 \\
\hline
\end{tabular}

of CIA strategies on the network topology and the density of connections. We assume ideal PHY and MAC layers: no packet is lost and no collision occurs. We assume a fixed radio range leading to random Unit-Disk Graphs (UDG). The results are presented with a confidence interval of $95 \%$.

Table II presents the percentage of maintained links. We show the average number of radio links: if two nodes have $r$ common channels, we count $r$ radio links. The measure is normalized according to the average number of neighbors in the single channel case. We consider an average density of 10 (number of neighbors in the single channel case) with nodes having 3 interfaces and 8 channels $\left(I_{v_{1}}+I_{v_{2}}<\mathcal{C}\right)$. For Mixed Interface Assignment, we consider 1 static interface [12], [14]. We will discuss the results separately for each strategy.

\section{A. Static Interface/Common Channel Assignment}

Each node uses channel $i$ for its $i^{t h}$ interface. Let $N_{\text {links }}\left(v_{1}, v_{2}\right)$ denotes the number of multi-channel links (i.e. using different channels) between nodes $v_{1}$ and $v_{2}$. One link exists for each channel (and transitively for each interface). We obtain:

$$
N_{\text {links }}\left(v_{1}, v_{2}\right)=\min \left(I_{v_{1}}^{S}, I_{v 2}^{S}\right)
$$

In other words, any pair of nodes that would be a neighbor in the single channel network would also be a neighbor in the multi-channel case leading to a connected network. Besides, the number of common radio links with one neighbor equals the minimum of the number of interfaces of both nodes. We can note that the density of connections is maximum in Table II: the density of connections attains 300\% when each node has 3 interfaces. Therefore, the efficiency of neighbor discovery is maximum: the neighbor will always be discovered $(P(R)=1)$ after only one single hello $(E[T R]=0)$. If a node sends a hello, any neighbor will receive it discovering the corresponding source. Thus, the neighbor discovery process does not depend on the relation between the number of interfaces per node.

More contention and interference may occur on channels pre-defined in the CCS when the number of nodes increases [17]. In particular, when nodes have a different number of interfaces some pre-defined channels will be used by less nodes leading to less contention. Thus, this strategy does not fully distribute traffic uniformly over all channels.

We can notice that this strategy is optimal when the number of interfaces equals the number of available channels, which is seldom the case. On the contrary, the radio bandwidth is wasted if two nodes do not have the same number of interfaces.

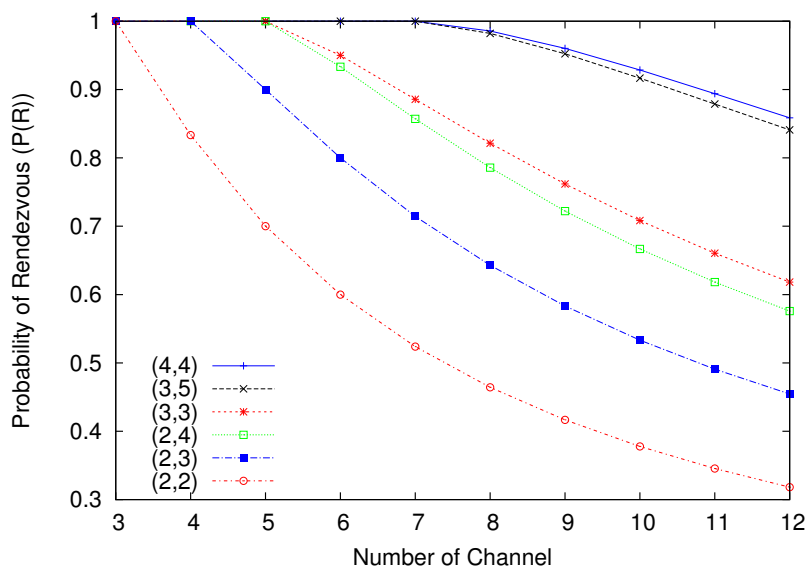

Fig. 1. Strategy Static Interface/Pseudo-Random Channel Assignment each curve is denoted by $\left(I_{v_{1}}^{S}, I_{v_{2}}^{S}\right)$.

In particular, each node must have as many interfaces as at least one of its neighbors.

\section{B. Static Interface/Pseudo-Random Channel Assignment}

One way to reduce contention when all interfaces are static is to apply Pseudo-Random Channel Assignment (Section III-B2) to static interfaces. However, the network is more likely to be disconnected and with less available links than under Static Interface/Common Channel Assignment strategy. It presents the lowest connectivity (cf. Table II). In the same way, the length of routes (number of hops) may increase.

When the sum of interfaces of both nodes is strictly greater than the number of channels, at least one channel is common to two neighbors: $P(R)=1$ and $E[T R]=0$.

Otherwise, if $I_{v_{1}}^{S}+I_{v_{2}}^{S} \leq \mathcal{C}, P(R)$ and $E[T R]$ depend on the relation between the number of interfaces per node and the number of available channels. It results in a combinatorial problem of unordered samples without replacement. In this case, we can compute the rendezvous probability by means of Equation 4 when channels are selected at random:

$$
P(R)=1-\frac{\left(\begin{array}{c}
\mathcal{C}-I_{v_{1}}^{S} \\
I_{v_{2}}^{S}
\end{array}\right)}{\left(\begin{array}{c}
\mathcal{C} \\
I_{v_{2}}^{S}
\end{array}\right)} .
$$

Figure 1 shows the rendezvous probability as the number of channels increases. The number of orthogonal channels varies from 3 (as in IEEE 802.11b/g) to 12 (as in IEEE 802.11a). To examine the effect of the number of interfaces per node, we plot six different cases in Figure 1. Each case corresponds to a pair of numbers representing the number of interfaces of any two neighbors $v_{1}$ and $v_{2}:\left(I_{v_{1}}^{S}, I_{v_{2}}^{S}\right)$.

The results show that $P(R)$ depends more on the relation between the number of channels and the sum of interfaces than on the difference of the number of interfaces. When $I_{v_{1}}^{S}+I_{v_{2}}^{S}<<\mathcal{C}$, the network is more likely to be disconnected. When it is sufficiently close to $\mathcal{C}$, the network is more likely to be connected. Note that the probability of a rendezvous is locally optimal when the radio interfaces are uniformly 


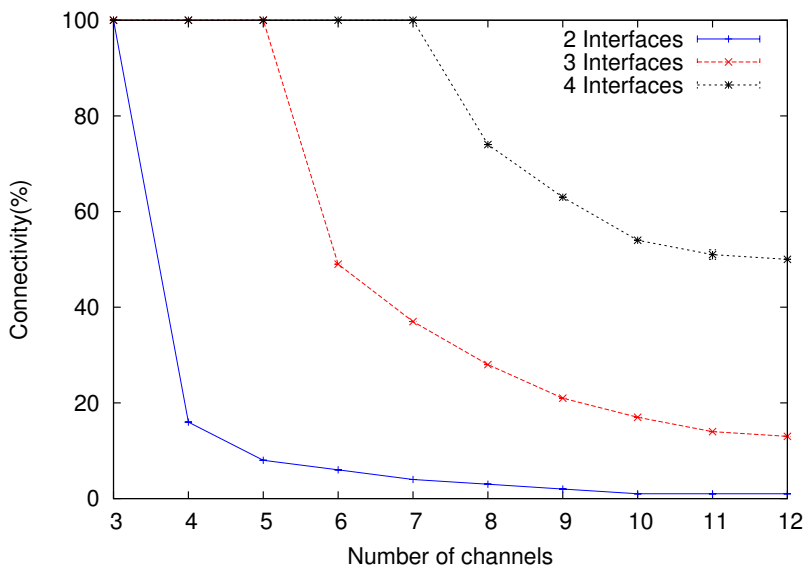

Fig. 2. Strategy Static Interface/Pseudo-Random Channel Assignment -300 nodes, average 10 single-channel neighbors

distributed between nodes. If a rendezvous occurs $E[T R]=0$, otherwise $E[T R]=\infty$.

We have also measured the impact of the number of channels on the network connectivity, i.e. the size of the largest component (cf. Figure 2). We can verify that the network is connected when the sum of the number of interfaces is strictly superior to the number of channels (i.e. one channel at least is common between both nodes). However, connectivity quickly decreases when the difference between the number of channels and the number of interfaces increases. According to Figure 1 , two nodes have a radio link with each other in $82 \%$ of the cases $(3+3$ interfaces, 8 channels). However, the global network connectivity is in this case only $28 \%$ (cf. Figure 2).

\section{Dynamic Interface/Adaptive Channel Assignment}

In this case, all interfaces are dynamic with Adaptive Channel Assignment. The main motivation is the use of all available channels to alleviate interference and channel congestion problems. However, the reassignment of dynamic interfaces in this strategy constantly alters the network topology and negatively impacts the network connectivity. Consequently, it presents the lowest connectivity similarly to Static Interface/PseudoRandom Channel Assignment (cf. Table II).

In this strategy, a rendezvous is important both for data exchange and neighbor discovery: two nodes can communicate if they have a channel in common. In particular, a pair of nodes with frequent rendezvous will be able to obtain a higher throughput.

The rendezvous probability depends on how channels are assigned except when $I_{v_{1}}^{D}+I_{v_{2}}^{D}>\mathcal{C}$, which implies $P(R)=1$ and $E[T R]=0$.

If $I_{v_{1}}^{D}+I_{v_{2}}^{D} \leq \mathcal{C}$, we assume that each node randomly chooses its own hopping sequence. We obtain the following probability of a rendezvous:

$$
P(R)_{k \mid t}=\sum_{p=k}^{t}\left(\begin{array}{c}
t \\
p
\end{array}\right)\left[1-\frac{\left(\begin{array}{c}
\mathcal{C}-I_{v_{1}}^{D} \\
I_{v_{2}}^{D}
\end{array}\right)}{\left(\begin{array}{c}
\mathcal{C} \\
I_{v_{2}}^{D}
\end{array}\right)}\right]^{p}\left[\frac{\left(\begin{array}{c}
\mathcal{C}-I_{v_{1}}^{D} \\
I_{v_{2}}^{D}
\end{array}\right)}{\left(\begin{array}{c}
\mathcal{C} \\
I_{v_{2}}^{D}
\end{array}\right)}\right]^{t-p},
$$

where $t$ is the number of channel switches done by dynamic

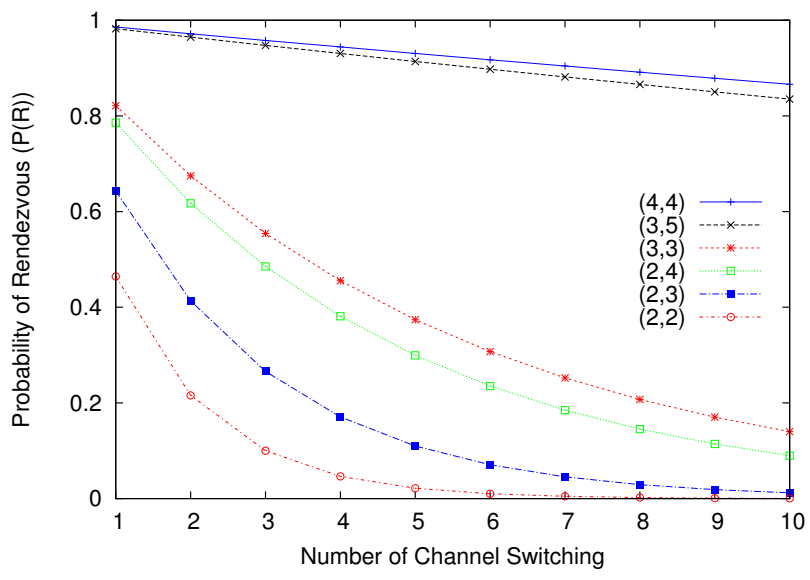

(a) All rendezvous are successful $(\mathrm{k}=\mathrm{t})$ - maximal throughput.

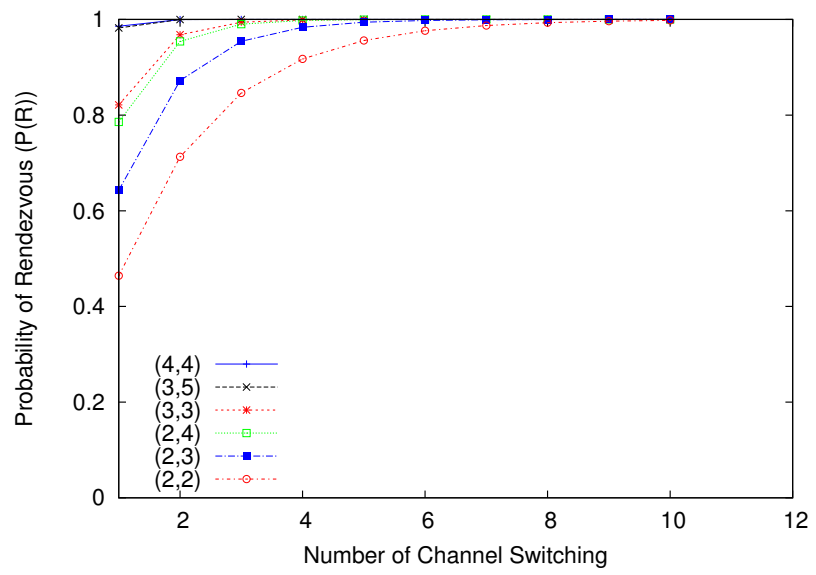

(b) 1 successful rendezvous $(\mathrm{k}=1)$ - neighbor discovery and at least one common 'slot'.

Fig. 3. Strategy Dynamic Interface/Adaptive Channel Assignments (8 channels).

interfaces and $k$ the number of a successful rendezvous between $v_{1}$ and $v_{2}$.

This random process is a sequence of Bernoulli trials since it is a sequence of $t$ independent repetitions. The number of channel switches is relative and not absolute: we count the cumulative number of pairs of channels explored by the nodes. If node $v_{1}$ switches its channel at $t$ and node $v_{2}$ at $t+\Delta_{t}$, we count 2 channel switches whereas if they change their channel at the same time, we count 1 channel switch. Thus, the channel switching process may or may not be synchronized.

If two nodes have $k$ common slots, they will take on average $\frac{t}{2 k}$ to discover each other:

$$
E[T R]=\sum_{k \in[1 . . t]} \frac{t}{2 k} P\left(R_{k \mid t}\right)+P\left(R_{0 \mid t}\right) * \infty=\infty
$$

Figure 3(a) presents the probability that a pair of nodes is always connected after each channel switching (i.e. the radio link always exists). Clearly, the radio link is mostly intermittent when the number of channel switches increases and the number of interfaces decreases.

We have also represented the probability that a pair of nodes has at least one rendezvous after $t$ switches (cf. Figure. 3(b)): 
the nodes can communicate at least once during channel scheduling. As expected, $P(R)$ increases with the number of channel switches. In both cases, $P(R)$ presents a better result when radio interfaces are uniformly distributed between nodes. We could derive the global connectivity from $P(R)$ values to obtain the same type of results as for the Static Interface/Pseudo-Random Channel Assignment (Figure 2). Because of the lack of space, we have chosen not to represent them here.

Network partitions may be avoided if nodes agree on deciding on which channel(s) exchange data. Nodes may publish their hopping sequences to make a future rendezvous easier [11] or reserve a predefined channel during at least one slot per sequence time. DaSilva et al. reduced the expected rendezvous time with predefined sequences [10]. Nevertheless, the scheme still requires a synchronization mechanism.

\section{Mixed Interface/Common and Adaptive Channel Assign- ment}

Strategies with Mixed Interface Assignment combine the advantages of the static and dynamic interfaces. In particular, rendezvous is simplified through static interface(s) while maintaining the flexibility coming from dynamic interface(s).

Because of the common control channel with a dedicated interface, the network is always globally connected even if it is not synchronized. In Table II, $100 \%$ of the connections correspond to the static assignment (CCS). The remaining connections (10\% on the average for 3 interfaces and 8 channels) arise from dynamic behavior.

Likewise static interfaces presented in Section IV-A, CCS preserves connectivity in this strategy. Thus, $P(R)=1$ and $E[T R]=0$. Static interfaces in Mixed Interface/Common and Adaptive Channel Assignment can be seen as a guaranteed way to make agreements for data exchange.

A pair of nodes may use its static interfaces to negotiate the channel to use for data exchange. The main idea is to isolate control packets from data packets by dedicating a fixed channel to exchange RTS and CTS packets and to avoid the interference between control and data packets. Besides that, since nodes are tuned to the same channels on static interfaces, they can overhear all the agreements made by other nodes and avoid busy channels.

However, this strategy has also the same drawbacks as the CCS approach: static interfaces may quickly become a bottleneck or on the contrary, will waste resources that could have been used for data exchanges.

\section{E. Mixed Interface/Pseudo-Random and Adaptive Assignment}

To avoid the problem above, this strategy also combines static and dynamic interfaces with the difference that static interfaces have Pseudo-Random Channel Assignment as described in Section III-B2.

If a new node enters the network, it first assigns random channels to its static interfaces. Then, it scans all available channels through its dynamic interfaces. The transmitter has to use one of its dynamic interfaces to send its packets through one channel used by one of the static interfaces of the receiver. Since the dynamic interfaces are only used for transmissions, deafness never occurs. Therefore, a dynamic interface will meet all the neighboring static interfaces leading to $P(R)=1$. However, despite the fact that network connectivity is guaranteed, the density of connections is reduced compared to Static Interface/Common Channel Assignment strategy (cf. Table II).

A node has to find with its dynamic interfaces one neighboring static interface. If both nodes have common static channels, they discover each other immediately. Otherwise, a node has to scan all non-static channels $\left(\mathcal{C}-I_{v}^{S}\right)$ and to stop as soon as it finds the first static interface. Let $T_{\text {sense }}$ be the interval length during which each dynamic interface senses a channel. Thus, we obtain the average neighbor discovery time:

$$
E[T R]=\left\lceil\frac{1}{2} * \frac{\mathcal{C}-I_{v_{1}}^{S}}{I_{v_{1}}^{D} * I_{v_{2}}^{S}}\right\rceil * T_{\text {sense }} *\left(1-\frac{\left(\begin{array}{c}
\mathcal{C}-I_{v_{1}}^{S} \\
I_{v_{2}}^{S}
\end{array}\right)}{\left(\begin{array}{c}
\mathcal{C} \\
I_{v_{2}}^{S}
\end{array}\right)}\right)
$$

where function \lceil\rceil rounds to the upper integer. Therefore, the lower the number of channels $(\mathcal{C})$ to scan and the higher the number of dynamic interfaces $\left(I_{v}^{D}\right)$ to scan, the lower is $E[T R]$.

If static interfaces are not used in the bootstrapping phase, only dynamic interfaces may discover each other. This clearly leads to the strategy Dynamic Interface/Adaptive Channel Assignment (Equation 5) in which all interfaces are dynamic.

\section{DISCUSSION}

Table III presents an overall summary of Channel and Interface Assignment (CIA) strategies for Multi-Channel MultiInterface (MCMI) Wireless Mesh Networks (WMN). It provides the equations for the rendezvous probabilities: the network designer can select the most suitable solution to guarantee connectivity with a certain probability for a given network density and number of channels and interfaces. Furthermore, advantages and limitations of each strategy are presented.

While it is true that static approaches provide suitable stability for routing protocols without path changes, re-ordering, channel switches etc., on the other hand, these approaches do not efficiently distribute the load among all available channels. The inability to adapt interfaces under heavy load and interference variations can drastically reduce the overall network performance.

Dynamic approaches have the ability to cover channels with few interfaces, thereby offering the potential to balance the load over different channels, to minimize interference, and to improve the capacity under heavy load. However, dynamic approaches may alter the network topology: some links are created while other may disappear. These changes can impact upper layers, especially routing protocols.

To combine the advantages of both approaches, a mixed solution can be applied. While connectivity is preserved with static interfaces, flexibility is achieved with dynamic interfaces. Anyway, static interfaces lead to the channel bottleneck problem. Furthermore, the way of performing dynamic channel assignment is still a challenge. 
TABLE III

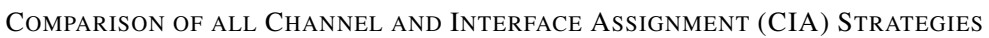

\begin{tabular}{|c|c|c|c|}
\hline Strategies & Connectivity & Neighbor Discovery & Assets \& Limits \\
\hline Static/Commom & Network is connected & $\begin{array}{l}P(R)=1 \\
E[T R]=0\end{array}$ & $\begin{array}{l}\ominus \text { multiple links between any pair of nodes } \\
\ominus \text { more contention and interference on predefined channels } \\
\ominus \text { bandwidth is wasted if nodes have different number of interfaces }\end{array}$ \\
\hline Static/Pseudo-Random & $\begin{array}{l}\text { Network can be parti- } \\
\text { tioned if } I_{v_{1}}^{S}+I_{v_{2}}^{S} \leq \mathcal{C}\end{array}$ & $\begin{array}{l}\triangleright \text { if } I_{v_{1}}^{S}+I_{v_{2}}^{S}>\mathcal{C} \\
P(R)=1 \\
E[T R]=0 \\
\triangleright \text { else } \\
P(R): \text { Equation } 4 \\
E[T R]=\infty\end{array}$ & $\begin{array}{l}\oplus \text { reduce contention and interference on predefined channels } \\
\ominus \text { lower connectivity } \\
\ominus \text { rendezvous is not always guaranteed due to deafness }\end{array}$ \\
\hline Dynamic/Adaptive & $\begin{array}{l}\text { Network can be parti- } \\
\text { tioned if } I_{v_{1}}^{D}+I_{v_{2}}^{D} \leq \mathcal{C}\end{array}$ & $\begin{array}{l}\triangleright \text { if } I_{v_{1}}^{D}+I_{v_{2}}^{D}>\mathcal{C} \\
P(R)=1 \\
E[T R]=0 \\
\triangleright \text { else } \\
P(R): \text { Equation } 5 \\
E[T R]: \text { Equation } 6\end{array}$ & $\begin{array}{l}\oplus \text { all available channels are used } \\
\oplus \text { balances the load among channels } \\
\ominus \text { requires synchronization and a rendezvous if connectivity has to } \\
\text { be guaranteed }\end{array}$ \\
\hline $\begin{array}{l}\text { Mixed/Common and } \\
\text { Adaptive }\end{array}$ & Network is connected & $\begin{array}{l}P(R)=1 \\
E[T R]=0\end{array}$ & $\begin{array}{l}\oplus \text { all available channels are used } \\
\oplus \text { simplicity: common control channel for a rendezvous } \\
\ominus \text { contention on the control channel that will negatively impact } \\
\text { performance }\end{array}$ \\
\hline $\begin{array}{l}\text { Mixed/Pseudo-Random } \\
\text { and Adaptive }\end{array}$ & Network is connected & $\begin{array}{l}P(R)=1 \\
E[T R]: \text { Equation } 7\end{array}$ & $\begin{array}{l}\oplus \text { all available channels are used } \\
\oplus \text { nodes can establish a radio link in a dynamic way } \\
\ominus \text { network capacity is not maximized }\end{array}$ \\
\hline
\end{tabular}

All things considered, a key issue is how to build an efficient mapping between all available channels and the interfaces at every mesh router when key design issues are addressed together: connectivity, interference, throughput/latency, stability, channel switching delay, and fairness.

\section{CONClusions AND Future Work}

We have focused on the connectivity problem in Wireless Mesh Networks. We have presented a formal evaluation of the existence of a link between two nodes and the average time to discover each other. The analytical framework permits to quantify network connectivity and to study more finely the neighbor discovery process and its consequences on the WMN. It also provides guidelines for the WMN designer: he can choose the most suitable strategy to obtain the desired properties.

While we have focused on the connectivity problem, we now plan to consider the evaluation of network capacity. We will extend the formalization of the link existence: it only provides an upper bound on network capacity regardless of interference. We also aim at corroborating the results with simulations and experiments to verify if we obtain a similar behavior. Finally, we will study how to combine the strategies to guarantee network connectivity with a certain probability while maximizing the network throughput.

\section{ACKNOWLEDGMENTS}

This work was supported in part by the French Government (MESR) and by ANR (Agence Nationale de la Recherche) contract ELAN ANR-08-VERS-008.

[2] J. Mo, H.-S. W. So, and J. Walrand, "Comparison of multichannel MAC protocols," IEEE Transactions on Mobile Computing, vol. 7, no. 1, 2008.

\section{REFERENCES}

[1] C. Chaudet, D. Dhoutaut, and I. G. Lassous, "Performance issues with IEEE 802.11 in ad hoc networking," IEEE Communications Magazine, vol. 43, no. 7, 2005.
[3] M. Kodialam and T. Nandagopal, "Characterizing the capacity region in multi-radio multi-channel wireless mesh networks," in ACM MobiCom, 2005.

[4] J. Crichigno, M.-Y. Wu, and W. Shu, "Protocols and architectures for channel assignment in wireless mesh networks," Ad Hoc Networks, vol. 6, no. 7, 2008.

[5] W. Si, S. Selvakennedy, and A. Y. Zomaya, "An overview of channel assignment methods for multi-radio multi-channel wireless mesh networks," Journal of Parallel and Distributed Computing, vol. 70, no. 5, pp. 505-524, 2010.

[6] R. Draves, J. Padhye, and B. Zill, "Routing in multi-radio, multi-hop wireless mesh networks," in ACM MobiCom, 2004.

[7] A. Adya, P. Bahl, J. Padhye, A. Wolman, and L. Zhou, "A multiradio unification protocol for IEEE 802.11 wireless networks," in IEEE BroadNets, 2004.

[8] M. K. Marina, S. R. Das, and P. Subramanian, "A topology control approach for utilizing multiple channels in multi-radio wireless mesh networks," Computer Networks, vol. 54, no. 2, 2009.

[9] X.-Y. L. Xufei M. and S. K. Makki, "Static channel assignment for multi-radio multi-channel multi-hop wireless networks," in WTASA, 2007.

[10] L. A. DaSilva and I. Guerreiro, "Sequence-based rendezvous for dynamic spectrum access," in IEEE DySPAN, 2008.

[11] P. Bahl, R. Chandra, and J. Dunagan, "SSCH: slotted seeded channel hopping for capacity improvement in IEEE 802.11 ad-hoc wireless networks," in ACM MobiCom, 2004.

[12] J. So and N. H. Vaidya, "Multi-channel mac for ad hoc networks: handling multi-channel hidden terminals using a single transceiver," in ACM MobiHoc, 2004.

[13] Y.-C. Tseng, S.-L. Wu, C.-Y. Lin, and J.-P. Sheu, "A multi-channel mac protocol with power control for multi-hop mobile ad hoc networks," in IPDPS Workshop, 2001.

[14] P. Kyasanur and N. H. Vaidya, "Routing and interface assignment in multi-channel multi-interface wireless networks," in IEEE WCNC, 2005.

[15] Y. Ding, K. Pongaliur, and L. Xiao, "Hybrid multi-channel multi-radio wireless mesh networks," in IEEE IWQoS, 2009.

[16] C. Sarr, C. Chaudet, G. Chelius, and I. G. Lassous, "Bandwidth estimation for IEEE 802.11-based ad hoc networks," IEEE Transactions on Mobile Computing, vol. 7, no. 10, 2008.

[17] K. N. Ramachandran, E. M. Belding, K. C. Almeroth, and M. M. Buddhikot, "Interference-aware channel assignment in multi-radio wireless mesh networks," in IEEE INFOCOM, 2006. 